\title{
Hierarchical decline of the initiative and performance of complex activities of daily living in dementia
}

\author{
Clarissa M. Giebel, MSc ${ }^{1,2,}$, Caroline Sutcliffe, $\mathrm{MSc}^{3}$, and David Challis, $\mathrm{PhD}^{3}$ \\ ${ }^{1}$ School of Health Sciences, University of East Anglia, Norwich, UK \\ ${ }^{2}$ Division of Neuroscience and Experimental Psychology, The University of Manchester, Manchester, \\ UK \\ ${ }^{3}$ Personal Social Services Research Unit, The University of Manchester, Manchester, UK
}

${ }^{*}$ Correspondence should be addressed to: Clarissa Giebel, Queen's Building, School of Health Sciences, University of East Anglia, NR4 7TJ, Norwich, UK. Email: c.giebel@uea.ac.uk; Tel: +44 1603593259 


\section{Abstract}

OBJECTIVES: Whilst basic activities of daily living hierarchically decline in dementia, little is known about the decline of individual instrumental activities of daily living (IADLs). The objective of this study was to assess initiative and performance deficits in IADLs in dementia.

METHODS: 581 carers completed the revised Interview for Deteriorations in Daily Living Activities in Dementia 2 to rate their relative's everyday functioning.

RESULTS: Initiating and performing IADLs deteriorated hierarchically, whilst people with dementia were consistently most impaired in initiating using the computer and managing finances. Initiating preparing a cold or hot meal and managing finances were more impaired than their performance, whilst performing maintaining an active social life for example were more impaired than their initiative. CONCLUSION: Findings can help identify the severity of dementia by understanding deficits in initiative and performance. This study has implications for the development of targeted interventions depending on the stage of dementia.

Keywords: dementia; activities of daily living; carers

Word count: 3425 


\section{Introduction}

Dementia affects 46 million people world-wide ${ }^{1}$, around 850,000 in the UK alone ${ }^{2}$. In an ageing society, this number is rising, so that finding effective ways to manage the disease is an important element in national dementia strategies ${ }^{3,4}$.

One primary symptom is increased dependency in everyday activities. Although both complex instrumental activities of daily living (IADLs) and basic ADLs deteriorate in the early stages of dementia, people with dementia (PwD) experience greater levels of deficits with IADLs early on ${ }^{5,6}$. The majority of research focuses on global impairment in performing IADLs or ADLs ${ }^{7-10}$, although examining individual activities can provide a better overview of the hierarchy of decline ${ }^{11,12}$, and help in targeting assistance to PwD with specific activities. Recently, new scales have been developed, such as the Amsterdam IADL scale ${ }^{14}$ or the IADL Profile which can also be used in aging ${ }^{15}$, and comprise a greater number of activities. Recent research based on the Interview for Deteriorations of Daily Living Activities in Dementia (IDDD) ${ }^{16}$ has highlighted how individual activities are differentially impaired in mild dementia only ${ }^{17,18}$. Moreover, using the IDDD and its derivative, the revised IDDD2 (R-IDDD2), allows for another distinction across everyday activities, the comparison between initiating and performing an activity.

The investigation of initiative and performance deficits in dementia is limited. Previous evidence is inconclusive as to which stage of an activity shows greater deterioration, with mixed populations including mild to moderate dementia or solely mild dementia ${ }^{16,17,19,20}$. A recent study reported that people in the mild stage of dementia were poorer at initiating cleaning, doing repair work, and preparing a hot or cold meal, whilst being poorer at performing dressing and following current affairs ${ }^{5}$. In particular, preparing a hot meal, using the computer, and finance and medication management were most impaired. All these investigations were based on the Interview for Deteriorations in Daily Living Activities for Dementia (IDDD), or a more recent version, the revised IDDD2 (R-IDDD2). Considering the hierarchical decline of ADLs and IADLs ${ }^{21}$, without any separation between the activity stages, there is a clear evidence gap as to how initiative deficits vary across mild, moderate, and severe dementia, and at what stage they start developing.

The primary aim of this study was to explore the level of initiative and performance decline of individual IADLs across the stages of dementia in a large national sample. Little evidence exists on impairments of individual activities, particularly IADLs, with even less information on activity initiative. Based on evidence from mild dementia, it is hypothesised that initiating and performing activities vary significantly for some activities, such as following current affairs or preparing a hot or cold meal, although this trend is hypothesised to become smaller in the advanced stages with little to no variation due to greater levels of overall impairment. Improved knowledge on initiative and performance deficits across mild, moderate, and severe dementia can highlight the point at which interventions should target individual activities in those different stages, and can thus improve the focus of nonpharmacological treatments. 


\section{Method}

\section{Participants and recruitment}

Carers (18 years or older) of people at any stage and type of dementia living in the community were identified through memory clinics and community based mental health services at $10 \mathrm{NHS}$ sites. The study was adopted onto the National Institute for Health Research portfolio and registered on the Join Dementia Research Network, (a UK-based research register for PwD, carers, and healthy volunteers to participate in dementia research), to increase access to eligible carers. Data were collected between October 2015 and May 2016. In addition, the researcher (CG) attended six ethnically-diverse carer groups across England.

\section{Materials}

Everyday functioning was assessed with the R-IDDD2 ${ }^{5}$. The R-IDDD2 contains 17 initiative and 20 performance activities. Each activity can be rated on a scale from '0' to ' 4 ' (never; seldom; sometimes; often; always difficulties). On the performance scale, each impaired activity can be rated further based on three sub-tasks provided. For example, using the telephone can be broken down into 'confusion whilst calling someone', 'forgetting numbers previously known', and 'general difficulties using the telephone'. In addition, the R-IDDD2 contains questions about the duration of caring responsibility, in years and hours per day, as well as the duration of symptom presentation. This provides an estimate of the severity of dementia where no data are available on the PwD' level of cognitive functioning. The R-IDDD2 and its predecessor the IDDD are psychometrically sound ${ }^{5,20}$.

Carer well-being was measured using the General Health Questionnaire 12 (GHQ-12) ${ }^{22}$ and the Adult Carer Quality of Life Questionnaire (AC-QoL) ${ }^{23}$. The GHQ-12 measures recent psychological distress, which includes the ability to concentrate, confidence, and problem management. A maximum score of 36 can be obtained, with higher scores indicating greater psychological distress. The AC-QoL is a 40-item questionnaire that assesses eight areas of carer QoL, including support for caring, caring choice, and ability to care. Each item is rated as 'never', 'some of the time', 'a lot of the time' and 'always' by the carer, which equates to a score of between ' 0 ' and ' 3 ' depending on the question. Higher scores indicate a higher QoL.

The QoL of PwD was measured using the Quality of Life in Alzheimer's Disease Proxy version (QoL-AD) ${ }^{24}$. The QoL-AD comprises 13 items including social relationships, finances and mood. A maximum score of 52 can be obtained, with higher scores indicating better QoL.

\section{Procedure}

Ethical approval was obtained through proportionate review by the Bristol NRES Committee South West (Ref: 15/SW/0271) prior to the study. Staff at the Trusts either distributed the questionnaire with a freepost return envelope to carers of PwD; or sent the questionnaire to the carers by post. If a pack 
was not returned within three weeks, staff contacted the carers to see if they required any assistance in completing the questionnaire or to answer any questions they may have. If carers had mislaid the pack, staff would send out a replacement.

The second mechanism of recruitment was via six dementia carer support groups. Carers completed the questionnaire after their written informed consent was obtained by the researcher (CG). Questionnaire completion took approximately 20 to 30 minutes.

\section{Data analysis}

Demographic characteristics were collected, and general information, scores on well-being scales and initiative and performance ratings of individual activities were calculated. These were analysed separately for mild, moderate, and severe dementia, and for the overall sample.

The severity of dementia was determined using data from a previous large European study of the transition of PwD living in the community into long-term care facilities, the RightTimePlaceCare programme ${ }^{25}$. This data-set which comprised 1,223 cases, classified PwD into mild, moderate, and severe dementia based on their score on the Standardised Mini-Mental State Examination ${ }^{26}$. A score of 24 to 20 indicated mild dementia, 19-10 moderate, and 9 to 0 severe dementia. These cut points have been utilised in other literature ${ }^{27}$. Frequency analysis and ANOVA with Bonferroni posthoc correction were employed to measure the ranges and significant variations in: symptom duration; hours of ADL care, hours of IADL care, and supervision across dementia severity. Single linear regression analysis was performed to analyse the level at which the four stated variables predicted dementia severity. ANOVA with Bonferroni correction showed significant variations in the duration of symptoms between mild and severe cases $(F(2,819)=5.810, p<.05)$; variations in hours of ADL care between all three stages $(F(2,1034)=33.456, p<.001)$; variations in hours of IADL care between mild and severe and moderate and severe dementia $(F(2,1035)=10.114, p<.001)$; and variations in hours of supervising the PwD between all three stages $(F(2,996)=19.405, p<.001)$. Single regression analyses showed that all four variables independently significantly predicted dementia severity.

The resulting data rules by which cases in the present study were categorised into mild, moderate, and severe dementia were initially tested on 30 cases in the RightTimePlaceCare data set. Categorising PwD in the present study relied primarily on data provided on hours of care, and also on the duration of symptoms, since recent literature ${ }^{28}$ shows a significant association between total caregiving time and dementia severity. Two researchers (CG, CS) independently rated all 604 cases of the present study, and those ratings were compared for inter-rater reliability using Cohen's ${ }^{29}$ kappa statistic. Any disagreements were discussed and a second rating was conducted. Any remaining disagreements in ratings were resolved through discussion.

Cohen's kappa statistics indicated good agreement (kappa $=0.655, p<.001)$ in the first, and very good agreement $(\mathrm{kappa}=.849, \mathrm{p}<.001)$ in the second rating round between the two independent reviews of the dementia stage. The remaining discrepancies were resolved through discussion to achieve a complete agreement. 
In the present data set, ANOVA, with Bonferroni post-hoc correction, was employed to analyse differences in the scores of the initiative and performance ratings, between the three cognitive stages of dementia. Paired samples t-tests were used to analyse within-group variations (mild, moderate, and severe dementia), in initiating and performing activities. The extent to which initiative deficits predict performance deficits was explored using linear regression modelling. Bivariate correlation analysis was used to calculate the associations between the GHQ-12, AC-QoL, and the QoL-AD with individual activities on the initiative and performance scales. Construct validity of the RIDDD2 was calculated using Cronbach's alpha.

Where the total score for an activity was missing, but scores on one or all three sub-activities were available, the median of the sub-activity scores was calculated for the overall activity score. For hours caring for ADLs, IADLs, or supervising, a maximum of 16 hours per day were employed where carers stated 24-hour care. This procedure was also used in previous published studies ${ }^{30}$. Statistical significance was set at $p<.05$ across all tests. All analyses were performed using SPSS Version 22.

\section{Results}

Overall, 604 carers completed questionnaires. Of these, 45 questionnaires were obtained through attending carer support groups, with attendance ranging from five to 10 carers per group. Of those recruited via NHS Trusts, 213 (35 per cent) were recruited via the Join Dementia Research Network. Of the total sample, 23 cases were excluded due to missing data for symptom duration and/ or number of hours of care, resulting in 581 cases in total. Using the previously specified severity ratings, 272 cases were classified as mild dementia, 170 as moderate and 139 as severe dementia.

Table 1 shows the demographic characteristics of the sample. In the overall sample, the majority of carers were female and White Caucasian, spouses or adult children, and on average 68 $(+/-11)$ years old. Carers tended to be the sole carers of the PwD and lived with them. The majority of PwD were male, White Caucasian, and on average 76 (+/-9) years old. The most prevalent type of dementia was Alzheimer's disease (52 per cent), followed by vascular dementia (14 per cent) and mixed dementia (16 per cent). On average, carers had noticed symptoms of the dementia for $4.4(+/-$ 3.0) years, whilst caring for the PwD for $2.9(+/-2.4)$ years. Carers spent the majority of caring on supervising their relative with dementia.

ANOVA with Bonferroni correction indicated that carer age was significantly lower in mild and moderate compared to severe dementia $(F(2,577)=8.262, p<.001)$. There were significantly fewer single carers in the mild dementia group than in the moderate and severe group $\left(X^{2}=52.770, p<.01\right)$, and significant variations in the relationship with a larger proportion of children and fewer spouses caring for people with mild dementia $\left(X^{2}=24.267, p<.001\right)$.

Carer distress was significantly lower in mild dementia compared to moderate and severe dementia $(F(2,568)=20.594, p<.001)$. Carer $Q$ oL was significantly higher in mild dementia compared with moderate and severe dementia $(F(2,513)=12.315, p<.001)$. PwD QoL was rated by carers as 
significantly lower in severe dementia compared to mild and moderate dementia $(F(2,552)=15.414$, $\mathrm{p}<.001)$.

\section{[INSERT HERE TABLE 1]}

\section{Impairments in initiating daily activities}

Table 2 shows the level of impairment in initiating everyday activities across mild, moderate, and severe dementia. In mild dementia, the proportion of PwD impaired in initiating an activity (thus either scoring 1, 2, 3, or 4) ranged between 47 percent (dressing) and 92 per cent (using the computer). Using the computer and handling finances were impaired to the largest extent both in the mean ratings and in the proportion of PwD impaired at this stage. Throughout the cognitive stages of dementia, these two activities remained amongst the most impaired activities. The ADL of dressing was the best preserved activity in mild, moderate, and severe dementia. At least 74 per cent of people with moderate dementia and 86 per cent with severe dementia experienced difficulties initiating any activity.

ANOVA with Bonferroni correction showed a significant gradual decline from mild compared to moderate and severe dementia for all activities except for finance management, which only significantly varied between the mild and moderate stage. For over half of the activities, PwD were poorer at initiating washing oneself, dressing, brushing hair/teeth, shopping, preparing a cold meal, cleaning house/repair work, following familiar routes, and following current affairs in the severe compared to the moderate stage. Figure 1 shows those activities that varied significantly across all three stages of dementia.

Single linear regression using the total sample $(\mathrm{N}=581)$ showed that initiative deficits predicted between 12 and 60 percent of variance in performance deficits.

\section{[INSERT HERE TABLE 2 AND FIGURE 1]}

\section{Impairments in performing daily activities}

Table 3 shows the level of performance deficits across dementia severity. The proportion of people impaired in the mild stages ranged from 45 to 90 per cent, whilst at least 69 and 84 per cent struggled performing any activity in the moderate and severe stages, respectively. Using the computer, managing finances, and driving were impaired to the greatest extent from the mild stage onwards. A large number of activities were often impaired, such as maintaining an active social life, preparing hot meals, using the telephone, and managing medication or finances.

ANOVA with Bonferroni post-hoc correction showed that people with mild dementia were significantly better at performing all activities compared to those in the moderate and severe stages. People with severe dementia were significantly poorer at performing over half of the activities than people with moderate dementia, including washing oneself, preparing a hot drink, dressing, brushing hair/teeth, shopping, preparing a cold meal, cleaning/ repair work, maintaining an active social life, following familiar routes, recognising familiar faces, and monitoring own day. Figure 2 shows those activities that varied significantly across all three stages of dementia. 


\section{Differences in initiating and performing everyday activities}

In both mild and moderate dementia, paired samples t-tests showed that PwD were significantly poorer initiating preparing a cold meal $\left(\mathrm{t}_{\text {mild }}=4.719, \mathrm{p}<.001\right.$; $\left.\mathrm{t}_{\text {moderate }}=2.028, \mathrm{p}<.05\right)$, preparing a hot meal ( $\left.\mathrm{t}_{\mathrm{mil}}=4.509, \mathrm{p}<.001 ; \mathrm{t}_{\text {moderate }}=3.057, \mathrm{p}<.01\right)$, cleaning and doing repair work $\left(\mathrm{t}_{\text {mild }}=4.077, \mathrm{p}<.001\right.$; $\left.t_{\text {moderate }}=3.976, p<.001\right)$ and finance management ( $\left.t_{\text {mild }}=3.129, p<.01 ; t_{\text {moderate }}=2.672, p<.01\right)$ than performing, whilst being significantly poorer at performing dressing $\left(t_{\text {mild }}=-5.660, p<.001\right.$; $t_{\text {moderate }}=-$ 4.914, $\mathrm{p}<.001$ ), maintaining an active social life ( $\mathrm{t}_{\text {mild }}=-2.685, \mathrm{p}<.01 ; \mathrm{t}_{\text {moderate }}=-2.123, \mathrm{p}<.01$ ), and following current affairs ( $\left.\mathrm{t}_{\text {mild }}=-4.224, \mathrm{p}<.001 ; \mathrm{t}_{\text {moderate }}=-3.787, \mathrm{p}<.001\right)$.

In severe dementia, PwD had significantly greater difficulty in initiating finance management $(\mathrm{t}=2.980, \mathrm{p}<.01)$ and driving $(\mathrm{t}=2.249, \mathrm{p}<.05)$ than performing these activities. They also experienced significantly greater difficulty performing preparing a hot drink $(\mathrm{t}=-1.992, \mathrm{p}<.05)$, dressing $(\mathrm{t}=-5.123$, $\mathrm{p}<.001)$, washing/brushing hair or teeth $(\mathrm{t}=-2.339, \mathrm{p}<.05)$, and maintaining an active social life $(\mathrm{t}=-$ $2.632, p<.05)$ than initiating these.

\section{[INSERT HERE TABLE 3 AND FIGURE 2]}

\section{Scale attributes}

The R-IDDD2 showed high construct validity for the Initiative ADL (Cronbach $\alpha=.913$ ) and Initiative IADL (Cronbach $\alpha=.875$ ) subscale, and high construct validity for the Performance ADL (Cronbach $\alpha=.904)$ and Performance IADL (Cronbach $\alpha=.941$ ) subscale.

\section{Discussion}

This study is the first to highlight a hierarchical decline in the initiative and performance of IADLs in dementia, with findings based on a large representative sample of community-dwelling PwD. Previous research has shown how different basic ADLs deteriorate throughout the stages ${ }^{11,17,21}$, with bathing, dressing, and continence for example deteriorating early on. Although IADLs are known to deteriorate largely in the early stages of the disease ${ }^{31}$, comprehensive knowledge on the components of IADLS (initiative and performance) and their decline has been very limited to date ${ }^{19,20,32,33}$.

This IADL hierarchy is particularly notable for its initiative decline. Previous studies have shown that initiating activities is impaired in dementia, yet to what extent it declines had to date not been explored $16,17,19,20$. Particularly in the early stages, initiating certain activities (i.e. preparing a hot or cold meal, finance management) was found to be more strongly impaired than their performance, with significantly greater disparity between initiative and performance in the mild stage compared to the moderate and severe stage. Considering that people in the severe stage still appear to experience stronger initiative than performance deficits for some tasks (i.e. finance management), this suggests that assessing the initiative to perform tasks remains an important factor in the assessment of everyday functioning in dementia. The R-IDDD2 employed for this analysis corroborates previous 
reports of its good psychometric properties ${ }^{5}$, and is thus a suitable tool for the comprehensive investigation of individual IADLs. Whilst rarely included in the assessment process, this study clearly highlights how initiative should be a vital component of this process.

The decline of initiative ratings throughout the course of dementia also offers suggestions for possible non-pharmacological treatments. Because variations between initiative and performance are evident across all three severity stages, interventions need to target each separately. Previous evidence failed to compare how both types of the activity process vary ${ }^{20}$. This study now clearly suggests though that even in the more advanced stages of dementia, PwD might benefit from receiving either support for improving their initiative or targeted training to help perform an activity. Considering that all PwD in this study still lived in the community, including those in the severe stages, offering suitable interventions to alleviate any initiative and performance difficulties can be important in delaying admission to a long-term care facility ${ }^{34}$.

Lacking initiative to perform an activity can have several reasons, which are linked to how initiative might be improved. These include memory and executive function difficulties and more exogenous factors. In some cases, such as driving, PwD may have had to give up their licence due to difficulties in performance, which can be linked to deficits in executive function and visual attention ${ }^{35}$, thus, they no longer initiate the activity. In other instances, PwD may forget that they should perform certain activities, such as doing the laundry or keeping an appointment. Deficits in memory and other types of cognition, such as executive function, are some of the primary symptoms of dementia ${ }^{36,37}$, and so far only performing IADLs has been shown to be related to memory and other types of cognition ${ }^{38}$. If this is the reason for lacking the initiative to perform a task, providing memory aids offering cues could alleviate these difficulties. Moreover, for some PwD, depression may also affect their lack of initiative. With depression being a common comorbid disorder of dementia ${ }^{39}$, treatments to help improve everyday functioning might need to consider avenues such as psychosocial therapy also in order to address depression as one of the possible causes of poor initiative. Whilst everyday activities can be influenced by a variety of comorbidities and other external factors, including physical limitations and environmental limitations ${ }^{40,41}$, this mail out study already contained other questionnaires on carer and PwD well-being and quality of life. Therefore, no further questionnaires were included so as to not overburden the carer and to maximise the return rate. Indeed, all these factors may need to be considered when designing interventions to improve initiative.

In this study, dementia severity was based on a comprehensive method of analysis involving a large European data set for devising rules for classification into mild, moderate, and severe dementia. However, data were not collected on the level of cognitive functioning of the PwD, as carers would also not have been able to provide this information. This is a limitation of the study. With the range of some variables (years since symptom onset and daily hours of ADL and IADL care and supervision) overlapping in values between mild and moderate, and moderate and severe dementia, in some cases it was difficult to rate the levels of impairment and clearly distinguish between them. Using solely a cognitive tool for severity staging however might be limited and impractical for carers to self-complete, whilst using four variables on the level of care needs in the present study allows a more comprehensive classification. Thus, whilst having a clear cognitive score for each PwD would 
have allowed for a more methodological method of severity staging, the rules devised from the European data set were as methodologically sound and tested.

\section{Conclusions}

In summary, this study indicates the utility of the R-IDDD2 in assessing everyday functioning across dementia stages. This tool can be of direct use in clinical practice in aiding early recognition of subtle symptoms in the mild stages of the condition, as well in severity staging. This is because the R-IDDD2 not only offers a very comprehensive breakdown of individual activities in their performance, but also considers the ability to initiate them. Improved recognition of dementia and thus improved diagnosis rates are central to governmental policies and worldwide initiatives ${ }^{42,43}$. Future work should further explore how the R-IDDD2 can help in the differential diagnosis of dementia subtypes, in addition to distinguishing between Alzheimer's disease, vascular, and mixed dementia ${ }^{33}$. Considering that different subtypes deteriorate at different speeds ${ }^{44}$, it would be valuable to assess whether the same is the case for the initiative of daily activities.

\section{Conflict of interest}

None.

\section{Description of authors' roles}

C.G. designed the study, collected and analysed the data, and drafted the article. C.G. and C.S. rated the dementia cases for severity. C.S. and D.C. commented on versions of the article.

\section{Acknowledgements}

This article presents independent research supported by the National Institute for Health Research (NIHR), under its Programme Grants for Applied Research programme (Grant Reference Number: DTC-RP-PG-0311-12003), and forms part of the doctoral thesis of CG, and was also funded by the University of Manchester's School of Nursing, Midwifery and Social Work Pump Priming Award 2015. We wish to thank all carers for taking part in this research, and staff at the following NHS trusts for their support in recruiting participants: Southend University Hospital; Black Country Partnership; Surrey and Borders Partnership; Lincolnshire Partnership; Derbyshire Community Health Services; Lancashire Care; Leicestershire Partnership; Southern Health; and North Essex. We also wish to thank Division 4 Greater Manchester NIHR Clinical Research Network for recruiting participants to this study through Manchester Mental Health and Social Care NHS Trust. Our thanks also extend to the JoinDementiaResearch Network as well as the following carer support groups for allowing us to recruit carers through their networks: Crossgates, Later Life Living, Life Story Network, Together Dementia, LMCP Care Link, and Bury Carers Centre. 


\section{References}

[1] Alzheimer Disease International. World Alzheimer report 2015: The Global Impact of Dementia. London, UK: Alzheimer Disease International, 2015.

[2] Prince M, Knapp M, Guerchet M, McCrone P, Prina M, Comas-Herrera A, et al. Dementia UK: Update. London, UK: Alzheimer's Society; 2014.

[3] Department of Health. 2010 to 2015 government policy: dementia. London, UK: Department of Health, 2015.

[4] Engedal K. The Norwegian Dementia Plan 2015 - "making the most of the good days". Int J Geriatr Psychiatr 2010; 25: 928-930.

[5] Giebel CM, Challis D, Montaldi D. The newly revised Interview for Deteriorations in Daily Living Activities in Dementia (R-IDDD2): Distinguishing initiative from performance at assessment. Int Psychogeriatr, in press

[6] Mioshi E, Kipps CM, Dawson K, Mitchell J, Graham A, Hodges JR. Activities of daily living in frontotemporal dementia and Alzheimer disease. Neurology 2007; 68(24):2077-2084.

[7] DiCarlo A, Balderischi M, Lamassa M, Bovis F, Inzitari M, Solfrizzi V, et al. Daily Function as Predictor of Dementia in Cognitive Impairment, No Dementia (CIND) and Mild Cognitive Impairment (MCl): An 8-Year Follow-Up in the ILSA Study. J Alz Dis 2016; 53(2):505-515.

[8] Nagaratnam N, Nagaratnam K, O'Mara D. Bayer-Activities of daily Living Scale in Mild and Moderate Dementia of the Alzheimer Type. Am J Alzheimers Dis Other Demen 2013; 28(8): 784789 .

[9] Pocnet C, Rossier J, Antonietti JP, von Gunten A. Personality features and cognitive level in Patients at an early stage of Alzheimer's disease. Personality Individual Differences 2013; 54(2):174-179.

[10] Vermeersch S, Gorus E, Cornelis E, De Vriendt, P. An explorative study of the relationship between functional and cognitive decline in older persons with mild cognitive impairment and Alzheimer's disease. Br J Occ Therap 2015;78(3): 166-174.

[11] Delva F, Edjolo A, Peres K, Berr C, Barberger-Gateau P, Dartigues JF. Hierarchical structure of the activities of daily living scale in dementia. J Nutrition Health Aging 2014; 18(7): 698-704.

[12] Sclan SG, Reisberg B. Functional Assessment Staging (FAST) in Alzheimer's Disease: Reliability, Validity, and Ordinality. Int Psychogeriatr 1992; 4(3): 55-69.

[13] Lawton MP, Brody EM. Assessment of older people: Self-maintaining and instrumental activities of daily living. Gerontolol 1969; 9(3): 179-186.

[14] Sikkes SAM, de Lange-de Klerk ES, Pijnenburg YA, Gillissen F, Romkes R, Knol DL, et al. (2012). A new informant-based questionnaire for instrumental activities of daily living in dementia. Alzheimers Dement 2012; 8: 536-543.

[15] Bier N, da Cunha Belchior P, Paquette G, Beauchemin E, Lacasse-Champagne A, Messier C, et al The Instrumental Activity of Daily Living Profile in Aging: A Feasibility Study. J Alz Dis 2016; doi: 10.3233/JAD-150957

[16] Teunisse S, Derix MMA, van Crevel H. Assessing the severity of dementia. Arch Neurol $1991 ; 48(3): 274-277$.

[17] Giebel CM, Challis D, Montaldi D. A revised Interview for Deteriorations in Daily Living Activities in Dementia (R-IDDD) reveals the relationship between social activities and well-being. Dementia 2014; doi: $10.1177 / 1471301214553614$ 
[18] Giebel CM, Sutcliffe C, Challis D. Activities of daily living and quality of life across different stages of dementia: a UK study. Aging Ment Health 2015;19(1):63-71.

[19] Teunisse S, Derix MM. The interview for deterioration in daily living activities in dementia: agreement between primary and secondary caregivers. Int Psychogeriatr 1997; 9(1): 155-162.

[20] Voigt-Radloff S, Leonhardt R, Schutzwohl M, Jurjanz L, Reuster T, Gerner A, et al. Interview for Deterioration in Daily Living Activities in Dementia: construct and concurrent validity in patients with mild to moderate dementia. Int Psychogeriatr 2012;24(3):382-390.

[21] Reisberg B, Ferris SH, Anand R, de Leon MJ, Schneck MK, Buttinger C, Borenstin J. Functional staging of dementia of the Alzheimer's type. Annals New York Academy Scie 1984; 435: 481483.

[22] Goldberg D, Williams P. A user's guide to the General Health Questionnaire. Windsor, UK: NFER-Nelson, 1988.

[23] Elwick H, Joseph S, Becker S, Becker F. Manual for the Adult Carer Quality of Life Questionnaire (AC-QoL). London, UK: The Princess Royal Trust for Carers, 1998.

[24] Logsdon RG, Gibbons LE, McCurry SM, Teri L. Quality of life in Alzheimer's disease: Patient and caregiver reports. J Ment Health Aging 1999; 5(1):21-32.

[25] Verbeek H, Meyer G, Leino-Kilpi H, Zabalegui A, Rahm Hallberg I, Saks K, Soto ME, Challis D, Sauerland D, Hamers JPH, the RightTimePlaceCare Consortium. A European study investigating patterns of transition from home care towards institutional dementia care: the protocol of a RightTimePlaceCare study. BMC Public Health 2012;12: 68.

[26] Molloy DW, Alemayehu E, Roberts R. Reliability of a standardized mini-mental state examination compared with the traditional min-mental state examination. Am J Psychiatry 1991; 148(10): 102105.

[27] Earnst KS, Wadley VG, Aldridge TM, Steenwyk AB, Hammond AE, Harrell LE, Marson DC. Loss of financial capacity in Alzheimer's disease: The role of working memory. Aging, Neuropsychology, and Cognition 2001; 8(2): 109-119.

[28] Hajek A, Brettschneider C, Ernst A, Posselt T, Wiese B, Prokein J, et al. Longitudinal predictors of informal and formal caregiving time in community-dwelling dementia patients. Soc Psychiatry and Psychiatr Epidemiol 2016; 51(4):607-616.

[29] Cohen J. A coefficient of agreement for nominal scales. Educational Psychological Measurement 1960; 20(1): 37-46.

[30] Wübker A, Zwakhalen SM, Challis, D, Suhonen R, Karlsson S, Zabalegui A, et al. Costs of care for people with dementia just before and after nursing home placement: primary data from eight European countries. European J Health Econ 2015; 16: 689-707.

[31] Peres K, Helmer C, Amieva H, Orgogozo JM, Rouch I, Dartigues JF, et al. Natural history of decline in instrumental activities of daily living performance over the 10 years preceding the clinical diagnosis of dementia: A prospective population-based study. J Am Geriatr Soc 2008; 56(1):37-44.

[32] Bier N, Bottari C, Hudon C, Joubert S, Paquette G, Macoir J. The impact of semantic dementia on everyday actions: evidence from an ecological study. J Int Neuropsychol Soc 2013; 19(2): 162-172.

[33] Giebel CM, Burns A, Challis D. Taking a positive spin: Preserved initiative and performance of everyday activities across mild Alzheimer's, vascular and mixed dementia. Int J Geriatr Psychiatr 2016; doi: $10.1002 / g p s .4553$ 
[34] Afram B, Stephan A, Verbeek H, Bleijlevens M, Suhonen R, Sutcliffe C, et al. Reasons for Institutionalization of People With Dementia: Informal Caregiver Reports from 8 European Countries. J Am Med Dir Assoc 2014; 2:108-116.

[35] Whelihan WM, DiCarlo MA, Paul RH. The relationship of neuropsychological functioning to driving competence in older persons with early cognitive decline. Arch Clin Neuropsychol 2005; 20(2): 217-228.

[36] Schubert S, Leyton CE, Hodges JE, Piguet O. Longitudinal Memory Profiles in BehavioralVariant Frontotemporal Dementia and Alzheimer's Disease. J Alz Diseas 2016; 51(3):775-782.

[37] Levy G, Jacobs DM, Tang MX, Cote LJ, Louis ED, Alfaro B, Mejia H, Stern Y, Marder K. Memory and executive function impairment predict dementia in Parkinson's disease. Movement Disorders 2002; 17(6): 1221-1226.

[38] Monaci L, Morris RG. Neuropsychological screening performance and the association with activities of daily living and instrumental activities of daily living in dementia: baseline and 18- to 24-month follow-up. Int J Geriatr Psychiatry 2012; 27(2): 197-204.

[39] Saczynski JS, Beiser A, Seshadri S, Auerbach S, Wolf PA, Au R. Depressive symptoms and risk of dementia: The Framingham Heart Study. Neurol 2010; 75(1): 35-41.

[40] den Ouden MEM, Schuurmans MJ, Arts IEMA, van der Schouw YT. Association between physical performance characteristics and independence in activities of daily living in middle-aged and elderly men. Geriatr Gerontol Int 2012; 13(2): 274-280.

[41] Gitlin LN, Hauck WW, Dennis MP, Winter L. Maintenance of effects of the home environmental skill-building program for family caregivers and individuals with Alzheimer's disease and related disorders. J Gerontol Scie A: Biol Scie Med Scie 2005; 60(3): 368-374.

[42] Ministry of Social Affairs and Health. National Memory Programme 2012-2020: Creating a "Memory-Friendly" Finland. Helsinki, Finland: Ministry of Social Affairs and Health, 2012.

[43] Australian Government Department of Health. National Framework for Action on Dementia 20152019. Canberra, Australia: Department of Health.

[44] Mioshi E, Hsieh S, Savage S, Hornberger M, Hodges JR. Clinical staging and disease progression in frontotemporal dementia. Neurol 2010; 74(20): 1591-1597. 